\title{
RF Driven Currents in Fusion Devices
}

\section{Gormezano, Grenoble}

\author{
(Euratom - CEA Association, CEN)
}

Among the recent achievements leading to the development of a thermonuclear reactor is the generation of plasma currents, of hundred of kiloamperes over several seconds using RF waves, with a record figure of $300 \mathrm{kA}$. This implies that a tokamak system could be operated in a steady state or quasi-steady state, which is not possible when the plasma current is produced through a transformer coupling as at present. Success in maintaining the plasma current with RF waves with a good efficiency may, moreover, simplify some of the technical problems encountered in building a practical reactor based on nuclear fusion.

\section{Inductive Drive in a Tokamak Experi- ment}

In order to reach ignition, i.e. when the amount of heat generated by fusion reactions is sufficient to replace the external heating, the product of the ion density, $n$, and the energy confinement time, $\tau$ should exceed $6 \times 10^{19} \mathrm{~s} / \mathrm{m}^{3}$ in a thermalized deuterium-tritium plasma with a temperature in the range $5-10 \mathrm{keV}$. Tokamaks are the leading devices in the race to reach this criterion known generally as the Lawson criterion ${ }^{1}$ ). All magnetic confinement schemes use the fact that charged particles, which constitute a plasma, are forced to move parallel to a magnetic field, their orbits perpendicular to the field lines being limited in extent. In toroidal, or doughnut-shaped devices, confinement is obtained by bending the magnetic field lines into a circle. Unfortunately, such a magnetic configuration is not stable since forces are created that cause the particles to drift outwards. Such forces can be neutralized by twisting the magnetic lines of force ${ }^{2}$ ).

In a tokamak, this is obtained by combining a toroidal field encircling the doughnut hole, with a poloidal field en-

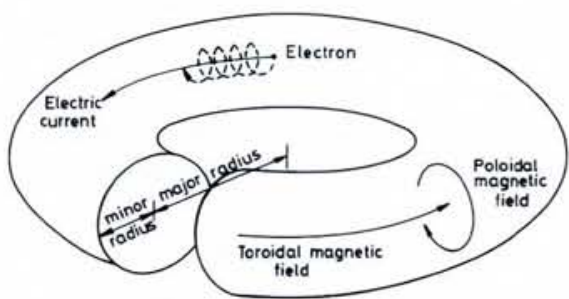

Fig. 1 - Schematic of magnetic fields in a tokamak configuration. circling the minor cross section as indicated in Fig. 1. Helical magnetic field lines are then formed which map out a set of nested magnetic surfaces. Hot plasma particles are confined to these surfaces. The toroidal field is readily produced by coils located outside the plasma which encircle the minor crosssection. The poloidal field is more difficult to obtain since it has to be produced by a toroidal current which, in practhe plasma acting as the secondary in a transformer circuit (Fig. 2). A central solenoid serves as the primary side with an air or iron core. A change in the magnetic field created by the transformer induces a toroidal electric field, $E$, according to Faraday's law:

$$
E \propto-\mathrm{d} B / \mathrm{d} t
$$

This field causes gas breakdown and "drives" a current into the plasma when the electron density builds up. But this electric field is established only so long as the magnetic field can be varied. Since the current in the poloidal coils cannot be increased indefinitely, such a tokamak is consequently a pulsed device, even though the pulse length can be maximized by running the coils from negative to positive current. A pulsed tokamak is susceptible to metal fatigue from the heat stress produced in a device which is hot when on and cold when off. In addition, the poloidal coil system is very complex and suffers from pulsed coil stresses and fatigue. For these reasons, a continuously operating tokamak is an attractive concept. Another advantage of a steady state systice, is supported by the plasma current,

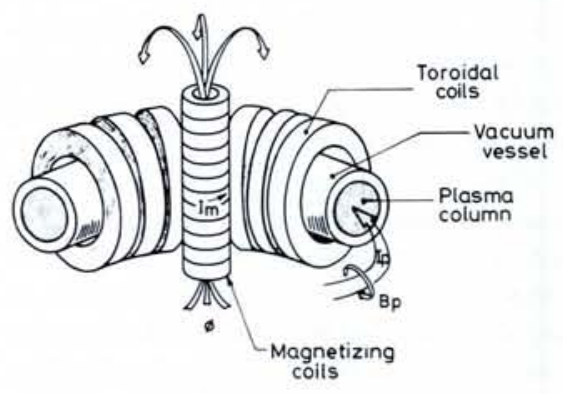

Fig. 2 - Schematic of a tokamak magnetic field system

tem would be to avoid repeated energy losses during each start-up.

Nevertheless, the inductive plasma current has proved to be very effective in providing the poloidal magnetic field required for particle confinement. It is the main characteristic of a tokamak since it not only provides the confinement, but also a source of heating for the plasma. The electric field produced by the change in the poloidal magnetic field pushes the plasma electrons as though they were a fluid. These collide with ions and other electrons and their energy is redistributed among the population. The resulting electron distribution can be considered as Maxwellian with an average drift velocity, $v_{D}$, in one direction. Electrons can thus be considered as a fluid with a parallel velocity, $v_{0}$ as indicated in Fig. 3a. The plasma is heated via the Joule effect (collisions with ions) and the "ohmic" power coupled to the electrons is a function of plasma current density, $j$, and plasma resistivity, $\eta$ :

$$
P_{\Omega} \propto \eta j^{2}
$$

Fig. 3 - Typical parallel electron velocity distribution and contours of constant $f$ on the $\left(v_{\perp}\right.$, $\checkmark$ /I plane for (a) a normal discharge and for (b) a run-away type discharge.

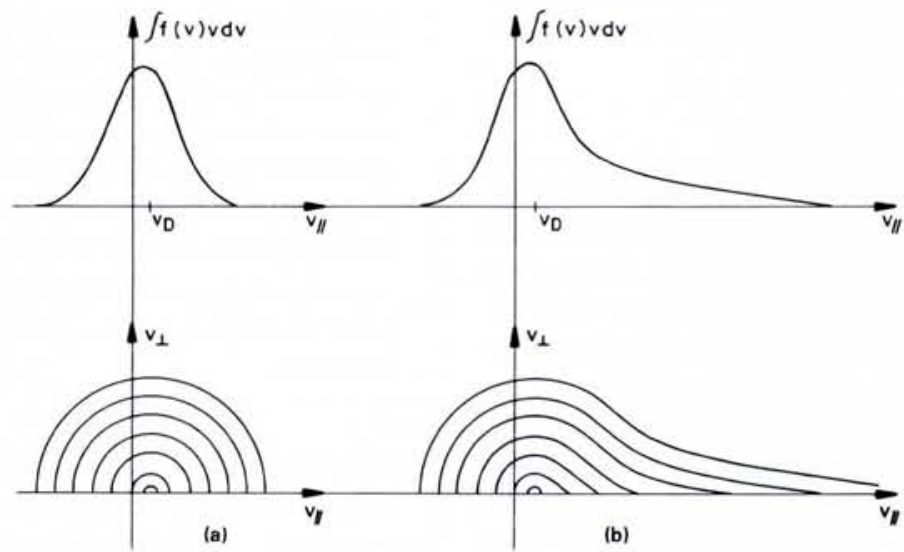


In practice, the plasma current is not always carried by the bulk electron population. The rate of collisions is a function of the plasma density and of the electron velocity:

$$
v_{\text {ee }} \propto n / v_{\mathrm{e}}^{3}
$$

Consequently, in a low density plasma, high energy electrons will have a low rate of collision and can gain more energy than they lose to the main electron population. These run-away electrons accelerate continuously giving rise to a distorted distribution as indicated in Fig. 3b. Such distributions occur when the field is above a critical electric field whose value depends upon the plasma parameters. The run-away electrons can reach very high energies (in the range of several $\mathrm{MeV}$ ) and carry a large part of the plasma current. Although plasma resistivity is decreased and a better use is made of the inductive drive, run-away discharges can cause severe damage to the tokamak device since electrons with too high an energy are not confined by the magnetic structure.

For normal (non run-away) discharges the plasma resistivity scales as:

$$
\eta \propto T_{\mathrm{e}}^{-3 / 2}
$$

and the ohmic power transferred to the plasma decreases as the plasma temperature increases; ohmic power alone is not sufficient for plasma ignition. Despite these limitations, impressive results have been obtained with induced plasma currents: the TFTR tokamak at Princeton has produced currents of 1.5 MA for $2 \mathrm{~s}$ giving $1.5 \mathrm{keV}$ plasmas at a density of $3.4 \times 10^{19} \mathrm{~cm}^{-3}$ and an energy confinement time of $0.25 \mathrm{~s}$ (Dec. 1983). The largest tokamak device, JET

\section{JET in AUGUST}

A little over one year after the first discharge, the toroidal magnetic field in JET was brought up to its design value of $3.4 \mathrm{~T}$ and subsequently the machine ran in hydrogen at a current of $3.6 \mathrm{MA}$ with a $4 \mathrm{~s}$ flat top.

On switching over to deuterium, the hydrogen content of the plasma fell rapidly to less than $10 \%$ and the following consistent results were obtained:

$\begin{array}{lr}\text { Plasma current } & 3.2 \mathrm{MA} \\ \text { Mean density } 3 \times 10^{19} \mathrm{~m}^{-3} \\ \text { Electron temp. } & 2.9 \mathrm{keV} \\ \text { lon temp. } & 2.0 \mathrm{keV} \\ \text { Confinement time } & 0.55 \mathrm{~s}\end{array}$

at Culham, achieved peak plasma currents of $3 \mathrm{MA}$ at the same time and set up 2.2 MA discharges lasting for $8 \mathrm{~s}$ with plasmas of $1.5 \mathrm{keV}$ at $n_{\mathrm{e}}=2.8 \times$ $10^{19} \mathrm{~m}^{-3}$ and an energy confinement time of $0.35 \mathrm{~s}$. Moreover, the performance of both devices is continuously being improved (see panel). In the high toroidal magnetic field Alcator-C at MIT $\left(B_{\mathrm{T}}=11 \mathrm{~T}\right)$, electron densities of $n_{\mathrm{e}}=$ $1.5 \times 10^{21} \mathrm{~m}^{-3}$ and an energy confinement time of $0.05 \mathrm{~s}$ have been produced by a plasma current of $0.5 \mathrm{MA}$. Although at a rather low temperature of $1.5 \mathrm{keV}$, the product $n \tau$ exceeds $6 \times 10^{19} \mathrm{~s} / \mathrm{m}^{3}$.

To overcome the drawbacks of inductive current drive as the main source of heating and confinement of a tokamak type reactor, many proposals have been made, with the main effort concentrating on additional heating. Very high temperatures, up to $7 \mathrm{keV}$, have been obtained via the injection of energetic neutral beams and wave plasma heating and big systems are being prepared for TFTR and JET as well as for the other large tokamaks. In contrast, a much smaller experimental effort has been devoted to the study of non-inductive current drive.

\section{Pushing Particles With Waves}

A great variety of waves can interact with a plasma. Among them, lowerhybrid waves, used for heating, have proved to be very effective for driving

\section{Cosa \\ EUROPEAN SPACE AGENCY \\ The European Space Agency has a number of vacancies in its Space Science Department (Astrophysics Division) as follows:}

1. Millimetre \& Sub-Millimetre Wave Astronomy (Staff position) Work in millimetre and submillimetre wave astronomy on the development of instrumentation for potential future space missions and to support studies on such missions.

Applicants should have experience in millimetre wave heterodyne observations of molecular clouds and have demonstrated research ability and a sound knowledge of heterodyne receivers. Ability to lead a small group in collaborative research with other European astronomers, to direct the development of advanced instrumentation for submillimetre wave astronomy from space, and to lead a study group examining the feasilibity of an 8 metre diameter space telescope for submillimetre wave astronomy.

2. Optical Astronomy (Research Fellowship)

The research activity centres around use of a prototype Faint Object Camera, developed for the Space Telescope in ground-based observations particularly exploiting its capability for narrow band imaging, spectroscopy and high time resolution studies. Preliminary development work in enhancements for this system and on second generation photon counting systems is under way.

Applicants should have a substantial research record in observational astronomy and be conversant with instrumental techniques and image data processing.

3. X-ray Astronomy (Research Fellowship)

Research and development of focal plane instrumentation for future generations of $\mathrm{X}$-ray imaging telescopes is being undertaken, based on the Gas Scintillation Proportional Counter development pioneered within the group. Observational data from the EXOSAT mission are being analysed.

Applicants should have research experience in the development of instrumentation, either in the space field or in nuclear physics and be conversant with associated electronic systems and data handling. Knowledge in astrophysics, while desirable, is not essential.

4. Gamma-ray Astronomy (Research Fellowship)

The group is heavily involved in the development of the Imaging Compton Telescope (COMPTEL) to be flown on NASA's Gamma Ray Observatory. Observational data from the COS-B mission are being analysed.

Applicants should have research experience in the development of instrumentation either in the space field, or nuclear physics, possess computational skills and be conversant with data analysis and image processing.

For all positions a Ph.D. or equivalent degree is required. The Staff position is a 4 year appointment and the research fellowship positions are normally available for up to two years.

An excellent knowledge of English or French is required with a working knowledge of the other language.

Applications should be directed to the Head of Personnel, ESTEC, Postbus 299, 2200 AG Noordwijk, the Netherlands, including detailed curriculum vitae and stating for which post(s) they wish to be considered. For enquiries phone 1719-83308. 


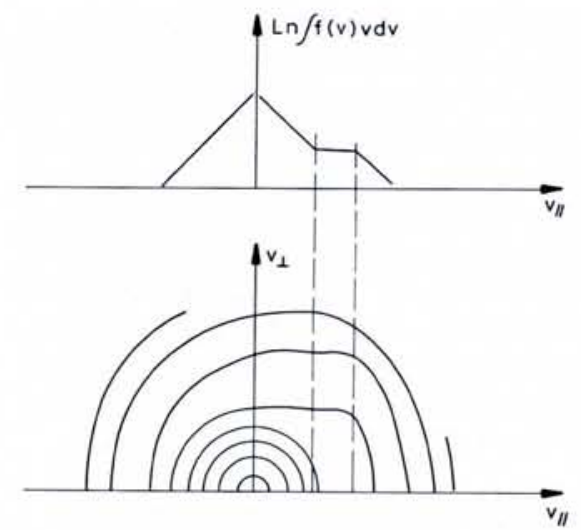

Fig. 4 - Typical parallel electron velocity distribution and contours of constant $f$ on the $\left(v_{\perp}, v_{1}\right)$ plane for a lower hybrid current drive discharge.

large plasma currents. First experiments were reported in 1980 from Japan and since then from the USA and Europe, following a theoretical proposal made by Nathaniel Fisch in $1978^{3}$ ).

The basic concept relies on the fact that a plasma is composed of oppositely charged particles, relative motion between which is an electric current. Waves can produce this relative motion but, they have to be launched in such a way that a class of particles stays in phase. Such resonant wave-particle interaction was first studied by Landau.

Pushing slow particles would seem to be most effective since the energy $\Delta E$ required to increase the momentum of a particle by $\Delta p$ is $\propto v \Delta p$. At first it was proposed to use waves of very low phase velocities such as Alfven waves (or even beams of neutral atoms) in order to accelerate these slow electrons ${ }^{4}$ ) but although it is very easy to transfer momentum to a slow electron, this momentum, or current, persists only so long as the electron does not collide with an ion. Hence, what matters is not the energy required to produce a given current so much as the power re- quired to sustain it. The rate $v_{\mathrm{ei}}$ of collisions of fast electrons with ions is of the same order as $v_{\mathrm{ee}}$ decreasing with velocity as $1 / v_{\mathrm{e}}^{3}$. With this in mind, Fisch proposed that fast electrons be used as current carriers and high frequency waves such as lower-hybrid waves used to push them.

Within the operating regime of experiments, the lower-hybrid wave dispersion relation can be written in the following simplified form ${ }^{5}$ ):

$$
\begin{aligned}
& \omega=\omega_{p e} k_{1} / k_{\perp} \\
& E_{\|}=E_{\perp} \omega / \omega_{\text {pe }}
\end{aligned}
$$

where $\omega_{\mathrm{pe}}$ is the plasma frequency $\left(\omega_{\mathrm{pe}}{ }^{2}\right.$ $\left.=n_{\mathrm{e}} e^{2} / m_{\mathrm{e}} \varepsilon_{\mathrm{o}}\right)$ and $E_{\|}, k_{\|}, E_{\perp}$ and $k_{\perp}$ are the electric fields and wave number components parallel and perpendicular to the magnetic field. When conditions are such that $\omega / \omega_{\mathrm{pe}}<<1$, the group and the phase velocities are directed almost parallel to the magnetic field. Resonant interaction occurs when the parallel phase velocity of the wave equals the parallel velocity of the electron:

$$
v_{\phi \|}=c / N_{1}=v_{l e} \text {. }
$$

$N_{\|}$being the parallel refractive index of the wave.

Theory describes the evolution of the electron distribution function, $f$, in the presence of a spectrum of RF waves by: $\frac{\partial f}{\partial t}=\frac{\partial}{\partial v_{\|}} \cdot D_{\mathrm{RF}}\left(v_{1}\right) \frac{\partial}{\partial v_{\|}} f+\left(\frac{\partial f}{\partial t}\right)_{c}$ where $D_{\mathrm{RF}}\left(v_{\|}\right)$is a diffusion coefficient induced by the RF electric field and $(\partial f / \partial t)_{c}$ is a collision term. The diffusion coefficient $D_{\mathrm{RF}}\left(v_{\|}\right)$is different from zero in the velocity range $\left[v_{1}, v_{2}\right]$ corresponding to the range of the parallel phase velocities of the injected waves. Its magnitude is proportional to the spectral energy density of the waves. If this is high enough, the wave-particle interaction results in the building of a plateau in the electron distribution function as shown in Fig. 4. This non-thermal distribution is very different from a run-away

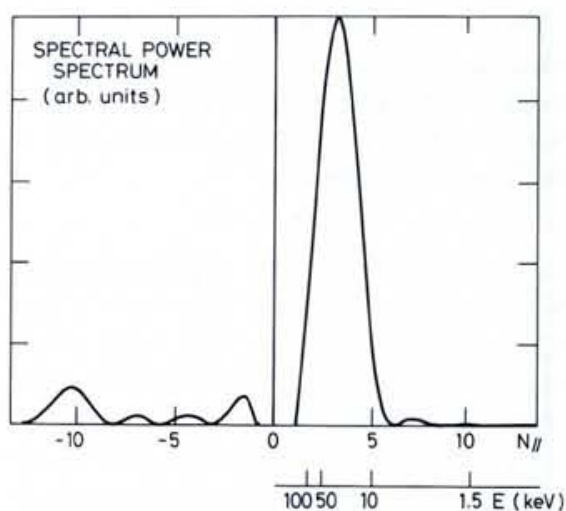

Fig. 5 - Typical parallel refractive index spectrum for a 6-waveguide grill.

distribution. It is to be noted that electrons cannot be accelerated continuously since the average $E$ field is zero during HF current drive. The energy of the RF electrons is bounded by an upper value corresponding to the highest phase velocity $v_{2}$. This is in contrast to a runaway distribution and this difference is well supported by experimental data.

\section{Demonstration of Current Drive}

Lower-hybrid waves can be produced with frequencies of the order of 1-10 $\mathrm{GHz}$. Slow electrostatic waves are launched into the plasma by an array of reduced section waveguides. The parallel phase velocity spectrum or its equivalent, the parallel refractive index spectrum, $N_{\|}=c / v_{\phi \|}$ can be shaped by adjusting the constant phase between individual waveguides. With a phase around $\pi / 2$, a progressive slow wave structure can propagate into the plasma. A typical parallel refractive index spectrum is reported in Fig. 5. The abcissa can be scaled with energies assuming resonance between wave and particle:

$$
E=m_{0} c^{2}\left[\left(1-1 / N_{\|}^{2}\right)^{-1 / 2}-1\right]
$$

As all the injected waves have a given frequency $\omega$, waves with negative $N_{\|}$ propagate in the direction opposite to

Fig. 6 (Below) - Long-pulse current drive on PLT. The plasma is formed by the $\mathrm{OH}$ system which was turned off at the current peak.

Fig. 7 (Right) - Current drive efficiency, $R I_{H F} / P_{H F}$, versus density from $P L T$, Petula-B and Alcator-C. Data are obtained for optimum conditions for current drive.
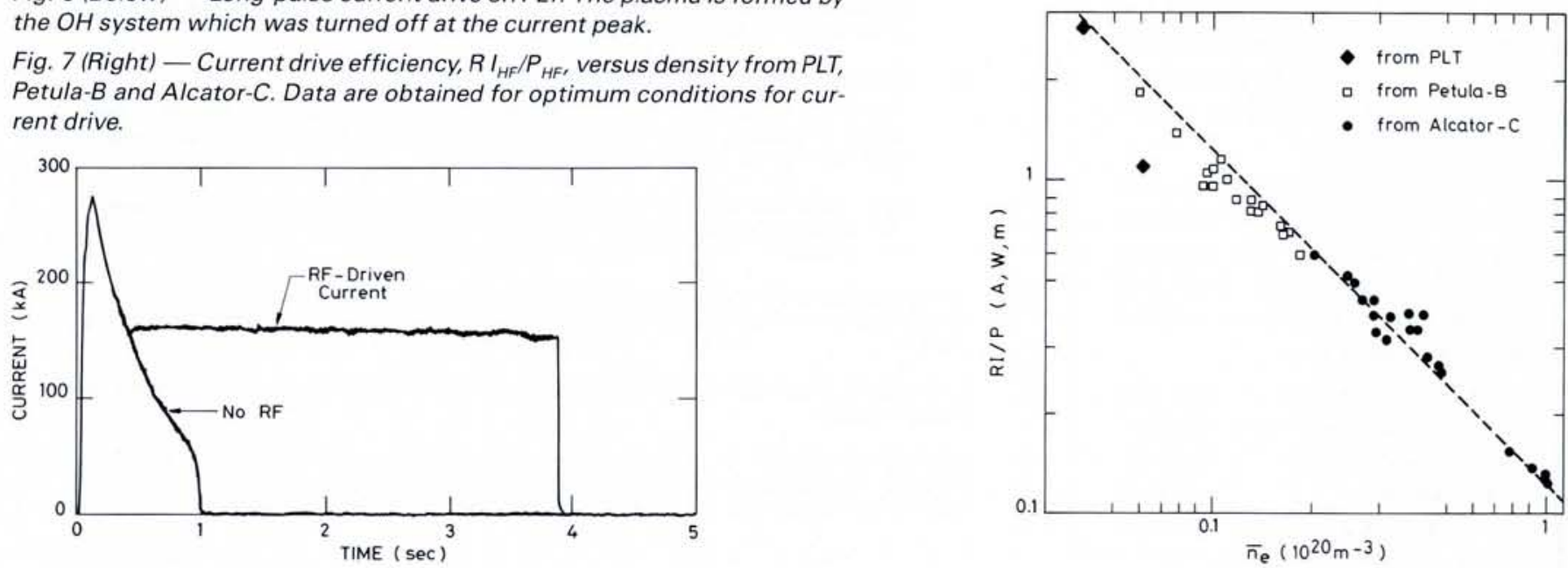
that of the waves in the main part of the spectrum. The energy contained, however, is not sufficient to drive a significant current. The spectrum can, as a result, be regarded as unidirectional.

First indications of non-inductive current drive were deduced from the derease in the $E$ field and a simultaneous decrease in the electron temperature when RF power was applied. In inductive discharges, such a decrease in the $E$ field is usually associated with an increase in electron temperature. In tokamaks using an iron transformer, the ohmic circuits cannot simply be deactivated and it was difficult in the earlier experiments on current drive to be sure that the current was being carried by suprathermal electrons (Fig. 4) and not by a run-away electron tail induced by a non-zero $E$ field (Fig. 3b). More convinc- ing evidence of non-inductive RF current drive was obtained when the plasma current was kept constant for times longer than the characteristic plasma current decay time, $L / R$, where $L$ is the self-induction and $R$ the resistance of the plasma column, in discharges with a zero DC electric field.

The Princeton PLT tokamak, with an air core transformer, has given a convincing demonstration of non-inductive current drive by operating the plasma discharge at a constant current over 3.5 $\mathrm{s}$, a time much longer than the usual magnetic drive duration. The corresponding data are shown in Fig. 6. The plasma is formed by the ohmic system which is turned off at the peak of the current. With $75 \mathrm{~kW}$ of RF power, the plasma current is maintained at a value of $160 \mathrm{kA}$. The plasma inductance is almost unchanged and negligible DC $E$ fields are measurable during the RF current drive period ${ }^{6}$ ).

Diagnostics measuring hard and soft bremsstrahlung $\mathrm{X}$-rays, millimeter microwaves at the Larmor gyration frequency and its harmonics, are able to give information on the shape of the electron velocity distribution, but theoretical and experimental work is still needed to deduce the real distribution from measured data. It appears that the velocity spectrum is bounded in the high velocity range.

Regardless of the mechanism invoked for generating suprathermal electrons, the amount of RF power required to sustain a current $I$ in a plasma of major radius $R$ for a plasma density $n_{\mathrm{e}}$ is:

$$
P_{\mathrm{RF}} \propto \ln n_{\mathrm{e}} R
$$

provided that these electrons lose their

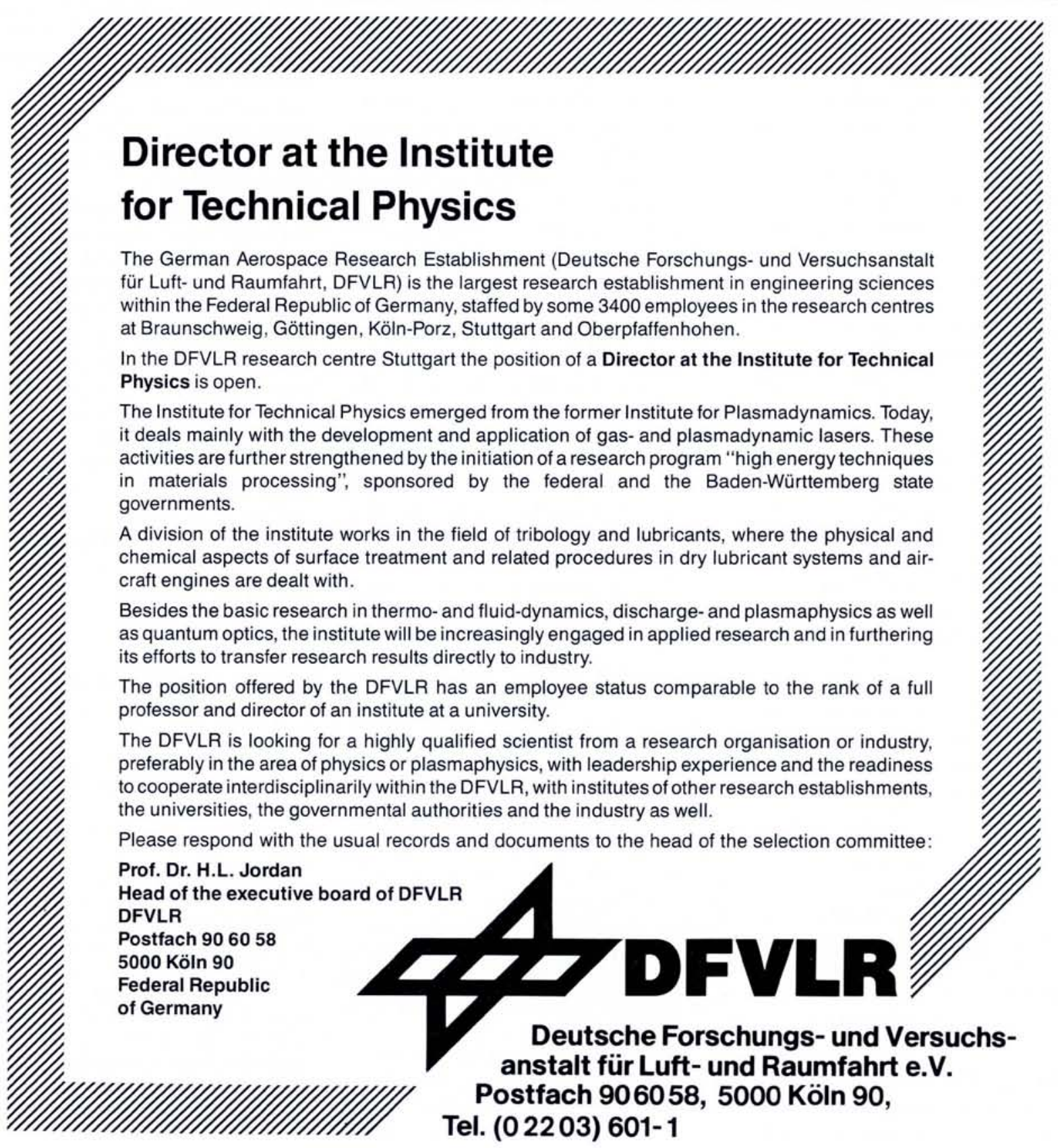


energy primarily by collisions with the bulk plasma. Data obtained from various experiments: PLT (Princeton), Alcator-C (MIT) and Petula-B (Grenoble) are shown in Fig. 7 in fair agreement with the above scaling law.

It is observed that the current drive efficiency is rather sensitive to the phase between wave guides determining the $N_{\|}$wave spectrum as would be expected from a consideration of the momentum transferred to electrons whose velocity is in resonance with the parallel phase velocity of the wave. It appears however in most experiments that this velocity range is about 5 to 15 times the thermal velocity of the bulk electron population.

From a theoretical point of view it is a mystery why waves with phase velocities in this range should drive a substantial current. If the electron distribution is assumed to be Maxwellian for $v_{\|}<v_{1}$ (Fig. 4) only a small fraction of the electrons will lie in the plateau region $\left[v_{1}, v_{2}\right]$ leading to a computed current that is smaller by orders of magnitude than the measured current.

A large amount of theoretical work is devoted to understanding physical mechanisms able to bridge the gap between the electron thermal velocity and the phase velocity of the lower hybrid waves. Possible candidates are the effect of a small amount of residual DC E field during current drive, instabilities driven by the large $T_{l} / T_{\perp}$ ratio for suprathermal electrons, non-linear effects shifting the wave spectrum, reflection of lower hybrid waves from the plasma surface, etc. It is to be noted that there is less uncertainty on the upper limit of the plateau $v_{2}$, which corresponds to the lowest value of $N_{1}$ which can propagate into the plasma (the accessibility condition). This condition, which depends upon the density and the magnetic field, may explain why the efficiency drops rapidly when the toroidal magnetic field decreases, as observed experimentally.

Another dramatic feature of lowerhybrid driven current, is the fact that the effect disappears completely above a certain density limit that is frequency dependent, Fig. 8. A common explanation for the density limit is related to the rapid growth of the perpendicular wave spectrum which can be effectively coupled to the plasma ion population. The fast ion tail so produced absorbs the main part of the RF power and the coupling to fast electrons disappears.

\section{Other Possible Mechanisms}

Waves other than lower-hybrid waves may also have a toroidal phase exceed- ing the electron thermal speed and so can be used to impart momentum to electrons. Examples of such waves are the magnetosonic waves and the ion cyclotron waves whose frequencies range from 0.1 to $1 \mathrm{GHz}$.

A rather different scheme for driving non-inductive currents has been proposed by Fisch and Boozer ${ }^{7}$ ). It consists of heating the plasma such that an asymmetric resistivity is created. A net electric current can be produced without the injection of any significant momentum parallel to the magnetic field if the ions move, on the average, in one direction and the electrons in the order. This can be achieved if electrons are preferentially heated when moving in one direction and consequently collide less with the ions in this direction. Electron cyclotron waves couple only to electrons moving in one direction (i.e. with one sign of $v_{\|}$ because of the Doppler resonance condition:

$$
\omega-k_{\|} v_{\|}-\Omega_{\mathrm{e}}=0
$$

where $\Omega_{\mathrm{e}}$ is the electron cyclotron frequency. In this wave-particle interaction, electrons gain perpendicular energy, i.e. $v_{\perp}$ increases, and collisions between ions and electrons are responsible for current generation, whereas electronelectron collisions tend to remove the asymmetry.

A similar asymmetry can be created by increasing the energy (principally in the perpendicular direction) of a particular group of resonant minority ions. The friction force on them being reduced, a relative drift is created between the minority ion species and the majority ion species and similarly with the electron species. This can be achieved by using ion-cyclotron waves. Experimental work for driving the current along these lines, as well as with direct injection of relativistic electron beams, is just starting.

\section{Future Lower-hybrid Wave Experiments}

When extrapolated to future large devices, lower hybrid current drive suffers from a decreasing efficiency with an increasing density and large radius. A complete steady-state large reactor tokamak would require too much power to drive the current. Present research is aimed at finding ways to improve that efficiency. Schemes where some level of $E$ field is always present, or where lowerhybrid current drive is used temporarily at low density while allowing the main transformer to be recharged are being considered.

Although other schemes for non-inductive current drive may have better possibilities for a thermonuclear reactor, lower-hybrid waves are so far the best

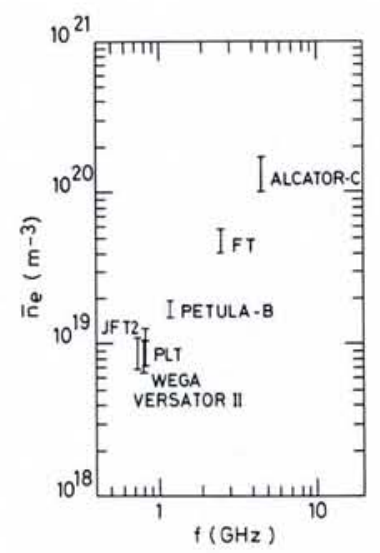

Fig. 8 - Critical density above which current drive disappears versus $R F$ frequency.

tool for studying a tokamak plasma at large currents without magnetic drive. First data from PLT and Petula-B indicate that both the energy confinement time and the stability are not degraded during lower hybrid current drive experiments and can even be improved. If such results are confirmed, they could be the main motivation for expanding the theoretical and experimental work which is presently going on.

The technique is sufficiently mature for lower-hybrid current drive to be envisaged as replacement for ohmic currents in large fusion devices. It is planned to extend the capability of the French tokamak Tore Supra (Cadarache) that is to be equipped with superconducting toroidal coils by using lower-hybrid current drive at a level of $1 \mathrm{MA}$ employing 8 $\mathrm{MW}$ of RF power at $3.7 \mathrm{GHz}$ for $30 \mathrm{~s}$. If successful, it is planned to operate the tokamak in a quasi-steady state at a slightly lower plasma current. This very challenging goal may demonstrate the full possibility of tokamaks with noninductive current drive.

\section{REFERENCES}

1. Lawson J.D.. Proc. Phys. Soc. B 70, 6 (1957).

2. Artsimovitch L.A., Nucl. Fusion, 12 (1972) 215 and Furth H.P., Nucl. Fusion, 15 (1975) 487.

3. Fisch N.J., Phys. Rev. Lett., 41 (1978) 873.

4. Ohkawa T., Nucl. Fusion, 10 (1970) 185.

5. Stix T.H., The Theory of Plasma Waves, (Mc Graw-Hill, New York) 1962.

6. See for instance: "Non Inductive Current Drive in Tokamaks". Proc. of the IAEA Technical Committee Meeting (1983) ed. D.F.H. Start, Culham Lab. Report CLM-CD (1983), and Proc. of the 11th Europ. Conf. of the European Physical Society Plasma Physics Division (1983), Plasma Physics, 26 (1984). 7. Fisch N.J. and Boozer A.H., Phys. Rev. Lett., 45 (1980) 720. 